\title{
Influence of indirect ultrasonic vibration on the microstructure and mechanical behavior of $\mathrm{Al}-\mathrm{Si}-\mathrm{Cu}$ alloy
}

\author{
H. Puga, J. Barbosa, S. Costa, S. Ribeiro, A.M.P.Pinto, M. Prokic \\ CT2M - Centre for Mechanical and Materials Technologies \\ Universidade do Minho, Azurém \\ 4800-058 Guimarães, Portugal
}

\begin{abstract}
The influence of high intensity ultrasound (US) propagating through a steel mold on the microstructure and mechanical properties of die-cast AlSi9Cu3, for different levels of electric power and at different distances to the waveguide/mold interface. The influence of those parameters on the morphology of $\alpha$ - $\mathrm{Al}$ and eutectic Si and on the volume of porosity were investigated and characterized. The morphological characterization revealed that the high intensity vibration not only promoted the formation of small $\alpha$-Al globular grains but also modify the eutectic silicon, as well as decreased the volume of porosity. Besides microstructure modification, US treatment improved the alloy mechanical properties, namely UTS and strain, which maximum values were $339 \mathrm{MPa}$ and $2.9 \%$ respectively by comparison to the values obtained for castings produced without US vibration. A mechanism of eutectic Si modification based on theoreticalexperimental analysis is proposed.
\end{abstract}

\section{Keywords}

Mechanical characterization, aluminum alloys, casting, nucleation 


\section{Introduction}

The performance of a mechanical component is often limited by the intrinsic characteristics of the used materials or disability/limitations inherent to the manufacturing process. Despite its widespread use, the casting process of aluminum alloys is not easy, since $\mathrm{Al}$ alloys are prone to heterogeneous structures, usually dendritic, and have a strong tendency to absorb hydrogen during melting, requiring suitable melt treatment to reduce and control the level of porosity and microstructure. Therefore, controlling the microstructure of Al-Si alloys is of primary importance to achieve high mechanical performance, thus requiring suitable degassing, refinement and modification.

Traditionally, degassing processes are based on the introduction of hydrogen free gas bubbles under pressure in the bath, for which the hydrogen atoms will diffuse and combine to form hydrogen gas, which is then expelled into the atmosphere [1]. The modification of the eutectic silicon phase is achieved by adding small amounts of strontium, antimony or sodium that promote the reduction in size and morphology change of silicon flakes in order to obtain a fibrous structure [2, 3]. Grain refinement is achieved by adding small amounts of titanium and/or boron [4] or by addition of Al-TiB [5] or Al-Ti-C [6] master alloys. However, the yield of these additions is low and there is no possibility to ensure a homogeneous distribution in the melt, which often results on very poor treatment efficiency.

During the last years an effort has been done to develop reliable ultrasonic techniques to control the microstructure of several engineering alloys, with particular emphasis to $\mathrm{Al}$ and $\mathrm{Mg}$ based ones, to overtake the problems associated to traditional refinement techniques. Khalifa et al. [7] and Jian et al. [8] demonstrated that it is possible to obtain non dendritic and globular grains of primary $\alpha$-Al phase smaller than $100 \mu \mathrm{m}$ by supplying acoustic energy to molten $\mathrm{AlSi} 7 \mathrm{Mg}$, isothermally and during solidification, respectively. Ultrasonic vibration can also be used to refine hypereutectic Al-Si alloys, as demonstrated by Feng et al. [9], who obtained equiaxed, $\alpha$-Al crystals of around 
$40 \mu \mathrm{m}$ and homogeneously distributed primary Si phase with average size of $180 \mu \mathrm{m}$ in AlSi23 alloy.

During the application of high intensity ultrasonic to a liquid metal, the alternate pressures, under certain conditions, may produce cavitation [10]. Nevertheless, in order to produce cavitation, it is necessary to verify a wide range of conditions which depend not only on the ultrasonic parameters and in the form they are transmitted to the medium, but also of the physical characteristics and the amount of impurities present in the melt [11]. In addition to high degassing efficiency caused by the development of cavitation [12] (evaluated by the hydrogen removal rate), the cavitation phenomena can also present a direct influence on the solidification mechanisms [11].

When a liquid metal is submitted to high intensity ultrasonic, the alternating pressure above the cavitation threshold creates numerous tiny bubbles in the liquid metal, which start growing, pulsing on a continuous expansion/compression regime and finally collapse. During expansion, the bubbles absorb energy from the melt, undercooling the liquid at the bubble-liquid interface, resulting in nucleation at the bubble surfaces $[8$, 11]. This mechanism is known by cavitation-enhanced heterogeneous nucleation. Another explanation to describe the cavitation-enhanced heterogeneous nucleation is based on the pressure pulse melting point, where the pressure pulse arising from the collapse of bubbles changes $T_{m}$ according to the Clapeyron equation. An increase in $T_{m}$ is equivalent to increasing the undercooling, so that an enhanced nucleation event is expected $[13,14]$. The first situation only occurs during the bubbles expansion stage, while the second needs the bubble collapse.

Moreover, bubbles collapse develops acoustic streaming throughout the melt, distributing the nuclei into the surrounding liquid producing a significant number of nuclei in the molten alloy. If a solid volume fraction is already present in the melt, the first dendritic grains can be broken and distributed in the liquid metal, acting as new nuclei. This mechanism is known by dendrite fragmentation [11].

Although the effect of cavitation in molten metal has been studied extensively, the mechanisms involved on the refining/modification of Al-Si alloys are not sufficiently studied, and references on this subject are not very clear. Besides, just a few works has 
been carried out on the effects of ultrasonic power on microstructure and corresponding mechanical properties of $\mathrm{Al}$ alloys. This paper presents and discusses a detailed study of the effect of ultrasonic power on the microstructure and mechanical properties of a AlSi9Cu3 alloy cast into permanent mold by applying ultrasound indirectly to the melt during the first stages of solidification.

\section{Experimental technique}

\subsection{Aluminum alloy}

The composition of the commercially available AlSi9Cu3 alloy used in this work was evaluated by Optical Emission Spectrometry and is presented in Table 1.

Tab. 1. Chemical composition of alloy

\begin{tabular}{ccccccccc}
\hline \multirow{2}{*}{ Alloy } & \multicolumn{8}{c}{ Chemical Composition (\%wt) } \\
\cline { 2 - 9 } & $\mathbf{S i}$ & $\mathbf{F e}$ & $\mathbf{M g}$ & $\mathbf{C u}$ & $\mathbf{M n}$ & $\mathbf{Z n}$ & Sn & Al \\
\hline AlSi9Cu3 & 9,15 & 0,66 & 0,18 & 2,25 & 0,26 & 0,47 & 0,10 & Bal. \\
\hline
\end{tabular}

\subsection{Experimental set-up}

Fig. 1 shows the experimental set-up used to perform ultrasonic grain refinement/modification. It consists on a novel MMM (Multi-frequency, Multimode, Modulated technology) US power supply unit developed by MP Interconsulting [15], a high power ultrasonic converter $(1200 \mathrm{~W})$, a $30 \mathrm{~mm}$ diameter and $150 \mathrm{~mm}$ long acoustic waveguide made of Ti6Al4V and the acoustic load itself which consists on a steel die (conical-trunk shaped with upper and lower diameter of 24 and $18 \mathrm{~mm}$, respectively, and $120 \mathrm{~mm}$ long) and the liquid metal. MMM technology is characterized by synchronously exciting many vibration modes through the coupled harmonics and subharmonics in solids and liquid containers. This technology produces high intensity multimode vibration that are uniform and repeatable, which avoid the creation of stationary and standing waves, so that the whole vibrating system is fully agitated, improving the refinement/modification process. 
The US power supply unit is fully controlled by dedicated Windows compatible software developed by MPI. Optimal US parameters: sweeping and $f_{\text {swm }}$ (frequency shift with modulation) for the selected resonance frequency and electric power are adjusted in order to produce the highest acoustic amplitude and the wide frequency spectrum in the melt, which is monitored with a specifically implemented feedback loop.

Besides the US refinement/modification unit, the set-up includes an electric resistance furnace equipped with a 0.5 litre $\mathrm{SiC}$ crucible, where $0.4 \mathrm{~kg}$ melting stocks of $\mathrm{AlSi}$ Cu3 were melted.
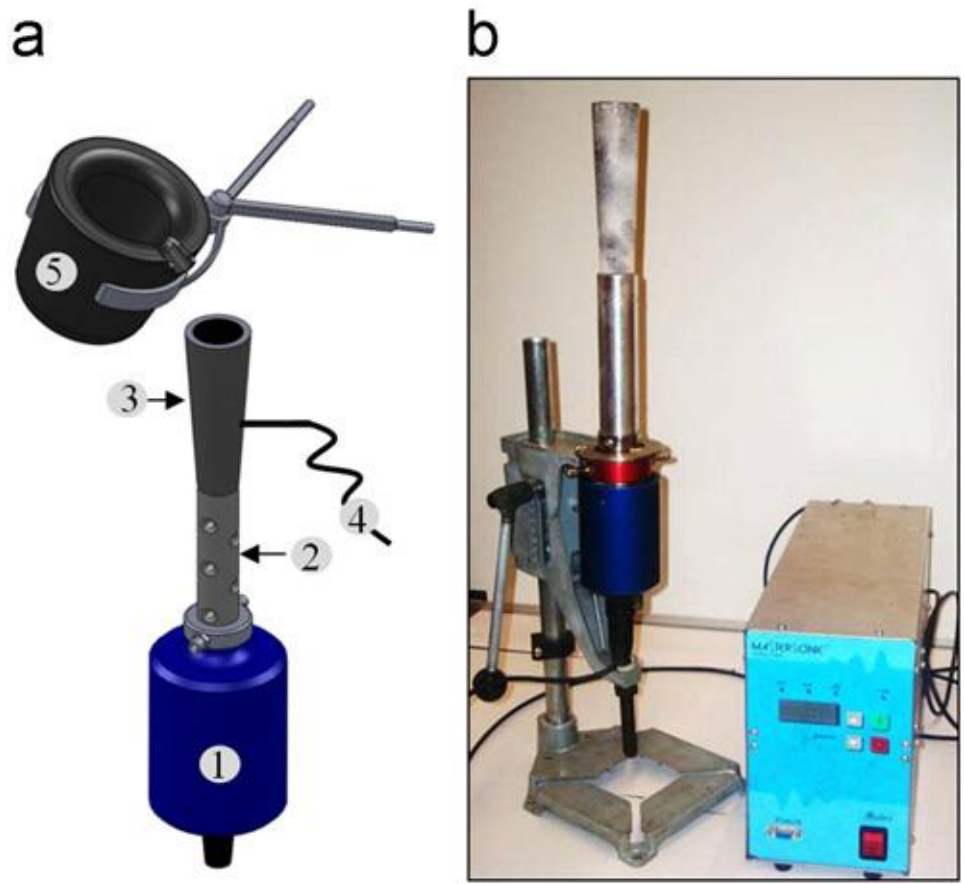

Fig.1. Schematic of the experimental set-up. (a) Conceptual model: 1- US converter, 2Waveguide, 3- Steel die, 4- Thermocouple, 5- Crucible, (b) Laboratorial unit.

\subsection{Experimental procedure}

The AlSi9Cu3 was melted and held inside the crucible at $700{ }^{\circ} \mathrm{C}$ during 15 minutes for homogenization. The molten alloy was then allowed to cool until the $680{ }^{\circ} \mathrm{C}$ and poured in a steel die pre-heated to $300{ }^{\circ} \mathrm{C}$. For every experimental condition, US vibration was applied continually between 680 and $580{ }^{\circ} \mathrm{C}$ melt temperatures, after which the US unit was stopped, and the melt cooled until room temperature. Melt temperature was 
controlled within an accuracy of $\pm 2{ }^{\circ} \mathrm{C}$. For the sake of comparison experiments were also carried out without ultrasonic vibration.

According to the alloy DSC curves [16], the range of melt temperatures were selected for continuous US processing. This procedure was used to evaluate the US capability to transmit acoustic energy to a continuously changing medium in what concerns to sound absorption due to the increase in viscosity with decreasing temperature.

Tests were performed using 200, 400 and $600 \mathrm{~W}$ electric power and $19.8 \mathrm{kHz}$ frequency using $\pm 0.25 \mathrm{kHz}$ sweeping. Four samples were cast for each experimental condition, three of them being used for mechanical testing and one for microstructure characterization.

For online motorization and acquisition of metal and steel die temperatures a LabVIEW based dedicated program was used together with a CompactDAQ (cDAQ) National Instrumetals system.

\subsection{Samples characterization}

Samples for microstructure characterization were taken from each cast sample by sectioning them perpendicularly to its longitudinal axis at distances to the interface waveguide/mold of $10 \mathrm{~mm}$ (Position A), $50 \mathrm{~mm}$ (Position B) and $90 \mathrm{~mm}$ (Position C). They were ground using $1200 \mathrm{SiC}$ paper and polished up to $1 \mu \mathrm{m}$. Those samples used for optical microscopy characterization were etched using Keller's reagent to reveal the resulting microstructure.

Optical microscope (OM) and Scanning Electron Microscope (SEM) with quantitative metallographic analysis capability were used to evaluate the shape and grain size of constituents. Image-ProPlus software was used to quantify the average grain size, average area of Si particles and the volume fraction of porosity.

For tensile testing, the specimens were machined from the as-cast samples according EN 10002-1:2004 with gauge length $L_{0}$ of $50 \mathrm{~mm}$ and cross section diameter $d_{0}$ of $10 \mathrm{~mm}$. Tensile tests were carried out at room temperature on a INSTRON - Model 
8874 testing machine using $0.5 \mathrm{~mm} / \mathrm{min}$ strain rate, to obtain yield strength, ultimate tensile strength and strain.

\section{Results and discussion}

\subsection{Effect of electric power of US on grain size}

In opposition to isothermal processing [16], on this work the acoustic radiator was replaced by the mold (indirect continuous processing), according to Fig. 1. Therefore, the propagation of acoustic energy to the melt is done directly through waveguide/mold interface, allowing pouring the melt at temperatures usually used on industry and more suitable to industrial practice implementation.

Fig. 2 shows the average $\alpha$-Al grain size for different levels of electric power at different distances to the waveguide/mold interface. It is clear that increasing electric power promoted higher grain refinement, and the best results were obtained for $600 \mathrm{~W}$. However, Fig. 2 also suggests that although the efficiency of grain refinement increased with increasing electric power, such increase was not linear and the reduction in grain size became less effective after reaching a certain electric power level. In Fig. 2 it can be noticed that 400 and $600 \mathrm{~W}$ curves are much closer than 200 and $400 \mathrm{~W}$ curves. Taking into consideration the $\alpha$ - $\mathrm{Al}$ grain size obtained at different distances to the waveguide/mold interface, a power increase from 200 to $400 \mathrm{~W}$ led to a decrease in grain size from 74 to $38 \mu \mathrm{m}, 75$ to $43 \mu \mathrm{m}$ and 95 to $51 \mu \mathrm{m}$, for the positions $\mathrm{A}, \mathrm{B}$ and $\mathrm{C}$, respectively. However, when electric power was increased to $600 \mathrm{~W}$, the grain size decreased on average 4 to $5 \mu \mathrm{m}$ for all positions.

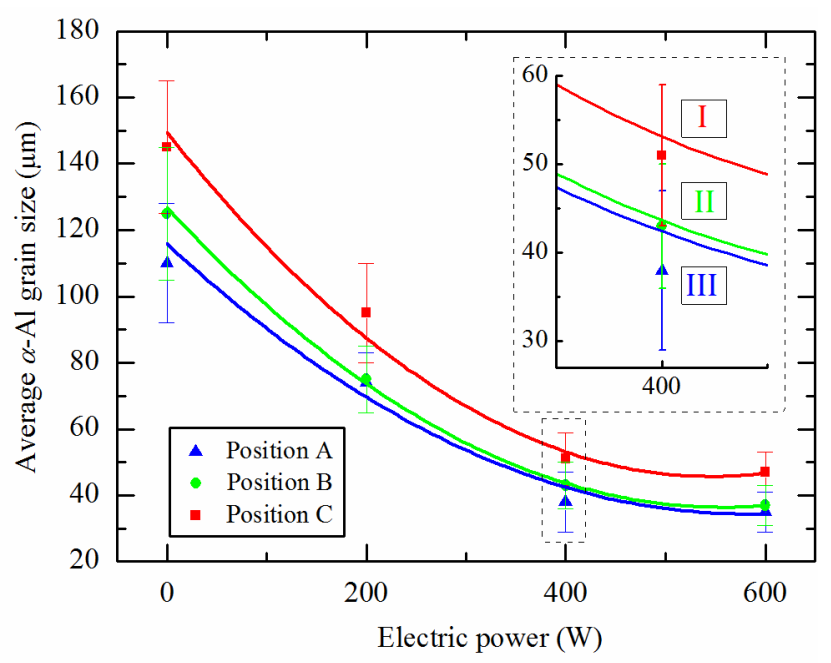

(a) 
Fig. 2. Effect of electric power of US on grain size of microstructure.

3.2 Effect of the distance to the waveguide/mold interface on the morphology of $\alpha$-Al grains

Fig. 3 shows the grain size and roundness distribution of primary aluminum grains of the AlSi9Cu3 alloy refined by US, at different distances to the waveguide/mold interface, for $400 \mathrm{~W}$ electric power. It is clear that with increasing distance to the interface, the medium size of $\alpha$-Al grains and the dispersion around that value increased (Fig. 3 (a)). Roundness of $\alpha$-Al grains trended to decrease in the same direction showing a wide dispersion of morphology. The worst result - 0.7 - was obtained for the largest distance to the waveguide/mold interface - Position C (Fig. 3 (b) - and is characterized by a mixture of globular and rosette-like grains, as presented in Fig.4.

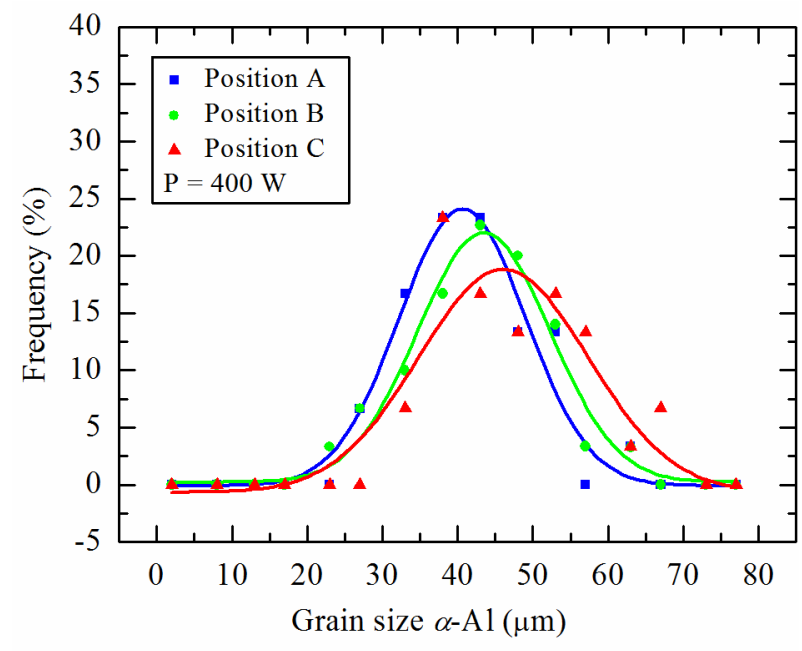

(a)

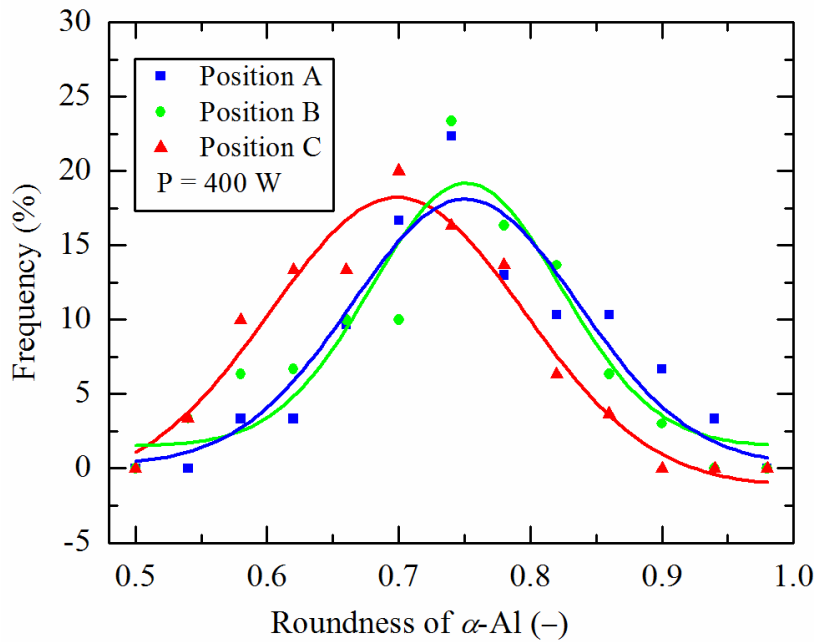

(b)

Fig. 3. Effect of distance to the waveguide/mold interface for $400 \mathrm{~W}$ electric power. (a)

Grain size distribution, (b) roundness distribution. 

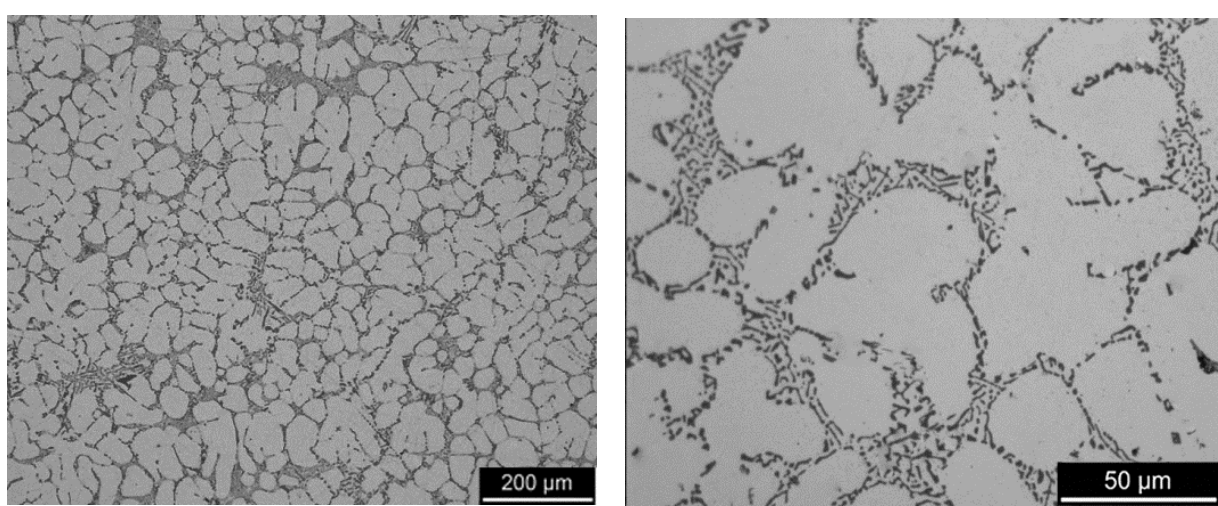

(a)
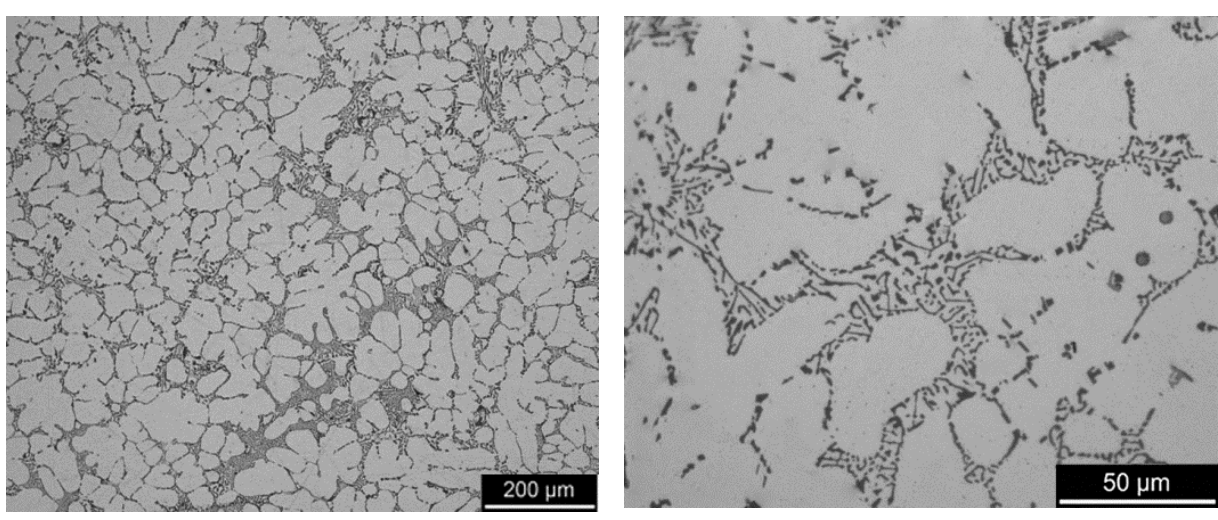

(b)
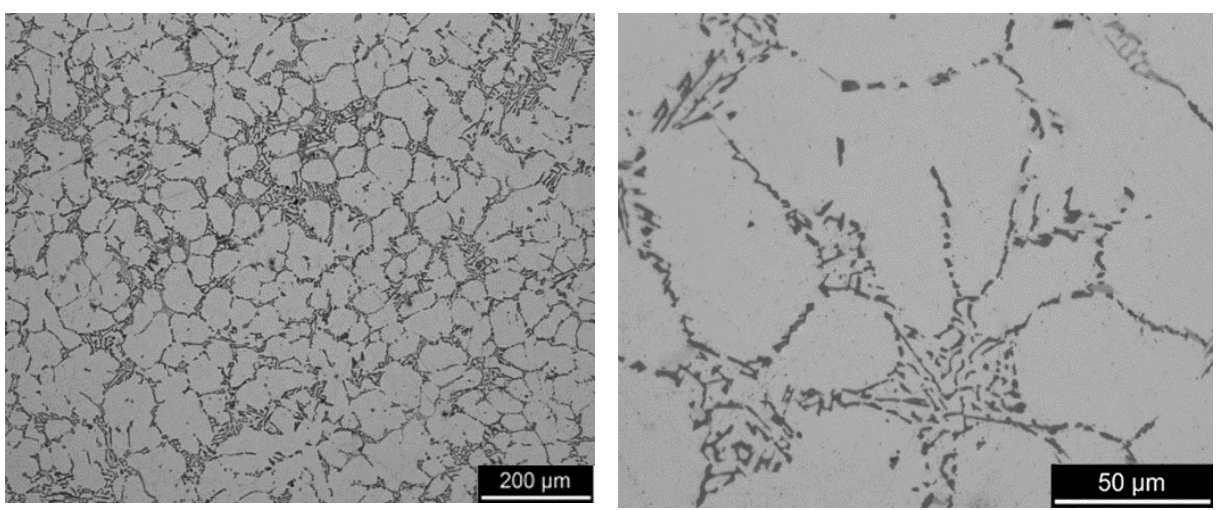

(c) 
Fig. 4. Microstructure of AlSi9Cu3 alloy processed by US using $400 \mathrm{~W}$ electric power at different distances to waveguide/mold interface. (a) Position A, (b) position B, (c) position $\mathrm{C}$.

The effect of power on the grain refinement efficiency can be explained as conjugation of two mechanisms: cavitation-enhanced heterogeneous nucleation and dendritic fragmentation. During the first stage of solidification - stage between pouring temperature and the liquidus temperature of the alloy - US grain refinement can only be attributed to cavitation-enhanced heterogeneous nucleation, since dendrite fragmentation would not be possible at those temperatures since solidification had not started yet. Below the liquidus temperature - corresponding to the formation of the first solid metal - cavitation can develop acoustic streaming caused by the collapse of bubbles in the remaining liquid promoting the fragmentation of dendrites that have already developed. The co-existence of these two mechanisms seems to promote a high density of nuclei in the melt, thus leading to the development of a large number of globular grains with small grain size.

The difference in $\alpha$-Al grains morphology, size and distribution registered at different positions - A, B and $\mathrm{C}-$ can be attributed to the effect of sound attenuation. Ultrasound loses its oscillation energy and sound intensity $(I)$ along the path $(x)$ in a melt, according to expression (1) [11]:

$$
I=I_{0} e^{-\propto x}
$$

If this loss is significant (it strongly depends on the viscosity of the melt), the intensity rapidly falls to the cavitation threshold and cavitation ceases, which causes a decrease in the number of cavitation bubbles. On the other hand, in the position nearest to the interface (Position A) the liquid cooling rate is higher than in the upper positions (Positions $\mathrm{B}$ and $\mathrm{C}$ ) resulting in increased viscosity of the melt in that area, which contributed to increase the attenuation coefficient $(\alpha)$ and, consequently, decreasing the acoustic intensity along the longitudinal axis of the sample. Thus, the effect of electric power in the final grain size is a consequence of the cavitation intensity and the number 
of cavitation bubbles induced at each power level that directly influences the number of solidification nuclei.

\subsection{Effect of indirect US processing on the morphology of eutectic silicon}

According to the microstructures shown in Fig. 4, it appears that the supply of acoustic energy to the melt during the stage of solidification has a high potential to change the morphology of eutectic silicon. In fact, the silicon lamellar/acicular morphology, characteristic of unmodified alloys, was transformed into a fibrous structure with rounded edges, compact and shaped polygon (Fig. 5), characteristic of modified alloys [3]. As verified with $\alpha$-Al grain refining, the potential of modification of silicon has tended to decrease with increasing distance of propagation of acoustic energy to the waveguide/mold interface, as it is evident from Fig. 6. For $400 \mathrm{~W}$ electric power, the average area of Si particles was 7, 9 and $12 \mu \mathrm{m}^{2}$ for positions A, B and C, respectively. Decreasing the value of the electric power to $200 \mathrm{~W}$, the average area of Si particles increased significantly (37, 47 and $52 \mu \mathrm{m}^{2}$, for positions A, B and C, respectively). The best results were obtained for $600 \mathrm{~W}$ electric power, for which the average area of silicon particles was approximately $2 \mu \mathrm{m}^{2}$ in all positions. The decrease in the modification efficiency of silicon in different areas of the samples can be attributed to the effect of sound attenuation as reported for the grain size in the same position.

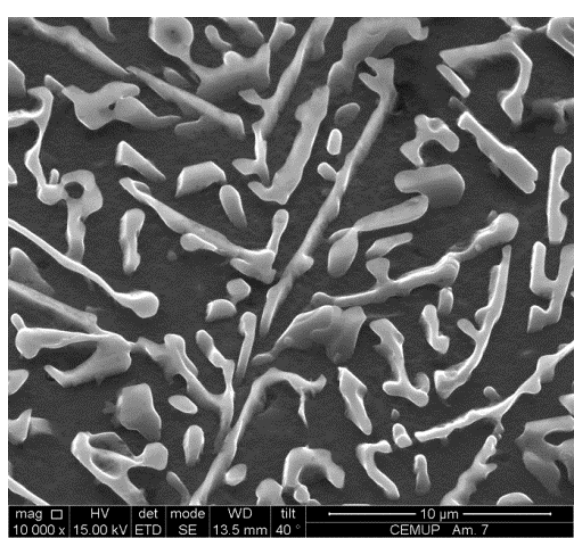

Fig. 5. Morphology of the eutectic Si particles at Position B obtained by SEM

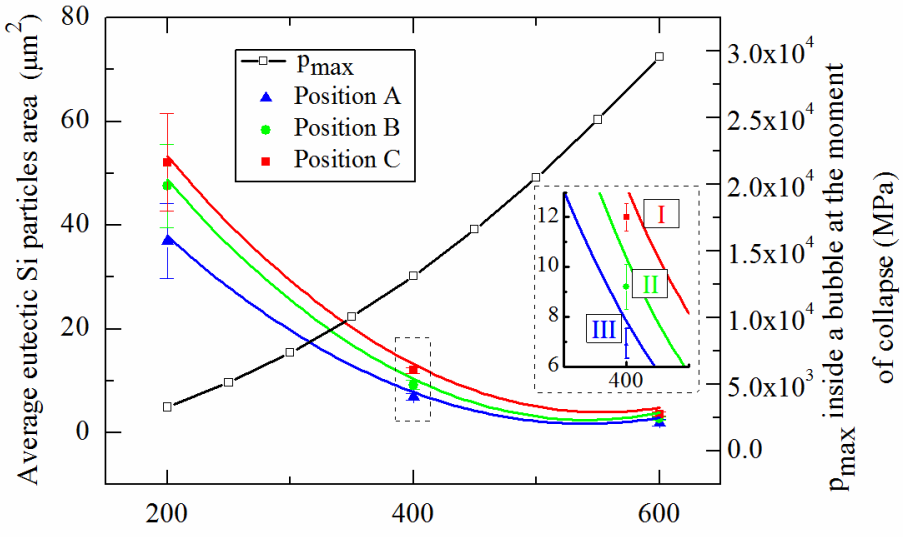


Fig. 6. Effect of US electric power on microstructure on the average area of eutectic silicon particles.

Unlike the refinement of the $\alpha$-Al grain, the mechanism of modification of eutectic $\mathrm{Si}$ during US processing of Al-Si alloys was not deeply studied yet. According to Jian et al. [17] two different features occur in samples processed by ultrasound: reduction of the eutectic phase spacing and a change in shape into a rosette-like form, that suggest that modification have occurred to a certain extent. On the other hand, Zang et al. [18] suggested, in a study on the effect of ultrasonic energy on the microstructure of A356 alloy, that the modification of silicon is a result of the fracture of silicon plates, since the shear stress applied on an element silicon area, $d S$, caused by the collapse of cavitation bubbles is higher than the value of the shear strength of same.

However by applying expression (2) proposed by Noltingk-Neppiras and Flynn to calculate the maximum pressure at the moment of collapse of a bubble inside a liquid, it can be shown that for the experimental conditions presented on this work, the fragmentation theory proposed by Zhang et al. [18] is not a viable explanation for the size reduction/modification of the silicon particles.

$$
p_{\max }=p \times\left\{\frac{p_{A} \times(k-1)}{p}\right\}^{k /(k-1)}
$$


where $p$ is the atmospheric pressure, $P_{A}$ the sound pressure calculated by equation

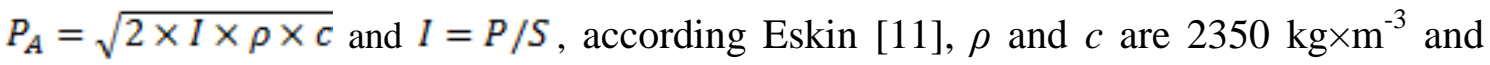
$5496 \mathrm{~m} \times \mathrm{s}^{-1}$ [19], respectively, $\mathrm{S}=3.14 \times 10^{-4} \mathrm{~m}^{2}$ and $k$ is the polytropic index of gas in the bubble ( $k=4 / 3$ for adiabatic pulsating [19]). By applying expression (2) for the electrical power values used on this work - 200, 400 and $600 \mathrm{~W}$ - the maximum pressure values at the moment of collapse of a cavitation bubble were $3.11 \times 10^{3}$, $1.24 \times 10^{4}$ and $2,80 \times 10^{4} \mathrm{MPa}$ (Figure 6), respectively, which are lower than the theoretical critical value of tensile stress for crack initiation in monocrystalline silicon $\left(3.00 \times 10^{4} \mathrm{MPa}[20]\right)$.

According to the presented results it is plausible that the mechanism of cavitation, in addition to have promoted refinement of the microstructure by heterogeneous nucleation/dendritic fragmentation, was also responsible for changing the eutectic silicon particles morphology. The development of cavitation caused by high acoustic intensity $\left(1.27 \times 10^{-6} \mathrm{~W} \times \mathrm{m}^{-2}\right.$ - calculated for an electric power of $\left.400 \mathrm{~W}\right)$ promoted a restriction to normal growth of silicon particles after their nucleation, according to Fig. 7. The cavitation bubbles can behave like atoms of chemical modifiers, promoting the modification of silicon through a mechanism similar to that observed in chemical modification. On the other hand, the decrease of temperature at the bubble/melt interface during the expansion phase of a cavitating bubble [14], suggests that cavitation also has influence on the nucleation frequency of eutectic cells remaining in the liquid, increasing and promoting a greater number of silicon particles nucleated.

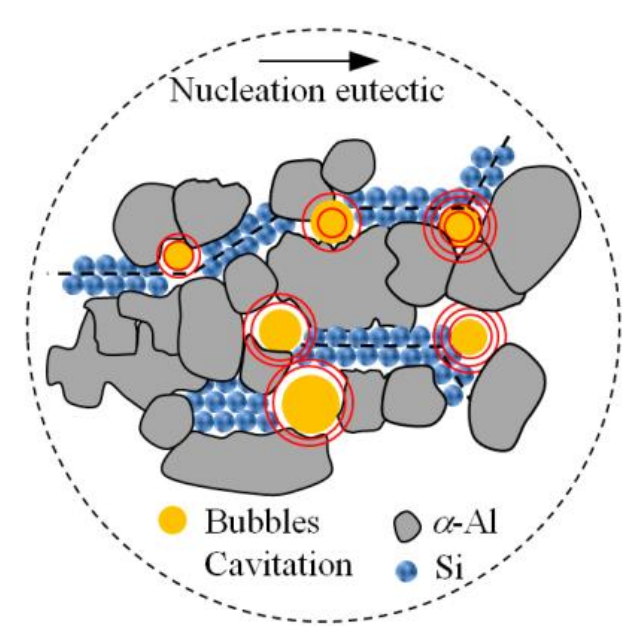


Fig. 7. Mechanism proposed for explain the eutectic silicon modification by US.

\subsection{Mechanical properties}

The ultrasonic treatment of AlSi9Cu3 clearly improved the alloy mechanical properties, especially in what concerns to ductility. From Fig. 8 it is evident that the ultimate tensile strength increased significantly with ultrasonic continuous processing. In those samples without US treatment, average UTS was $220 \mathrm{MPa}$. UTS increased to 270, 310 and 338 MPa after US processing at 200, 400 and $600 \mathrm{~W}$ electric power and melt processing temperature between 680 and $580{ }^{\circ} \mathrm{C}$. Besides UTS, an increase in strain was also verified. Strain increased from $0.6 \%$ in those samples without US treatment to $1.1,1.9$ and $2.9 \%$ after US processing at 200, 400 and $600 \mathrm{~W}$ respectively and melt processing temperature between 680 and $580^{\circ} \mathrm{C}$ (Figure 8).

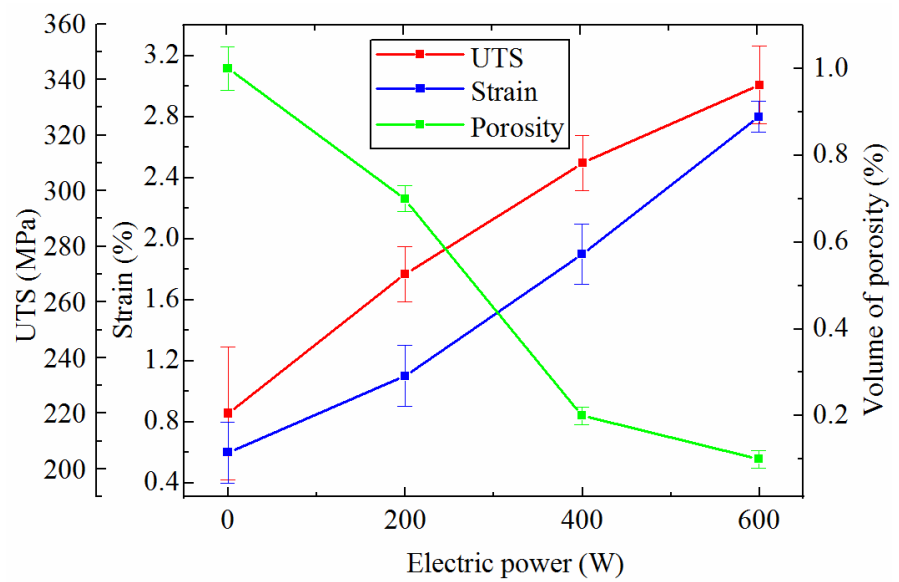

Fig. 8. Samples mechanical properties and volume of porosity for different levels of US electric power.

It is well known that mechanical properties of Al-Si alloys depend on several factors, with particular emphasis to microstructure morphology [21] and size and distribution of porosities [22]. In what concerns to microstructure, according to Panuskova et al. [21] and Gruzleski and Closset [1] the morphology of eutectic silicon is determinant to the 
mechanical properties. Although the size and morphology of the primary aluminum phase, as well as porosity distribution, can also play a significant role in the alloy mechanical behavior [16].

Porosity, one of the effects that can be attributed to the inadequate feeding associated to volumetric shrinkage of liquid on solidification and the decrease of hydrogen solubility in $\mathrm{Al}$ during cooling or even caused by chemical modification of eutectic silicon [1], is often present in $\mathrm{Al}$ castings. Besides microstructure refining and modification, US processing also have a great impact on the porosity of aluminum alloys [12]. From microstructures shown in Fig. 4, the samples sanity is evident since the level of porosity is very reduced (average porosity measured on positions $\mathrm{A}, \mathrm{B}$ and $\mathrm{C}$ ), and it has a tendency to decrease with increasing US power $-0.7,0.2$ and $0.1 \%$ after US processing at 200, 400 and $600 \mathrm{~W}$ electric power respectively as shown in Fig. 8. This is extremely beneficial with respect to mechanical properties of the alloys. The absence or small amount of porosity in alloys processed by US, can be attributed to the effect of acoustic cavitation developed in the liquid, in the first stage of solidification (higher temperatures that promoted hydrogen removal from the liquid phase, i.e., has degassing effect).

Taking into consideration the work results, UTS increase verified in our research can be attributed to the combined effect of the globular shape and small size of the $\alpha$-Al grain, the reduction of the volume fraction of porosity and the fine and short eutectic silicon fibers (Fig. 4 and 5). Although intermetallic particles have not been characterized, the US processed alloys didn't evidence significant presence of coarse and long particles like $\mathrm{Al}_{5} \mathrm{FeSi}$ (Figure 4) that are characteristic of this alloy, according to reports of Panuskova et al. [21] and Backerud et al. [23] and the microstructure of a sample obtained without US processing ( Fig. 9). Thus, its contribution to the UTS increase can't be excluded.

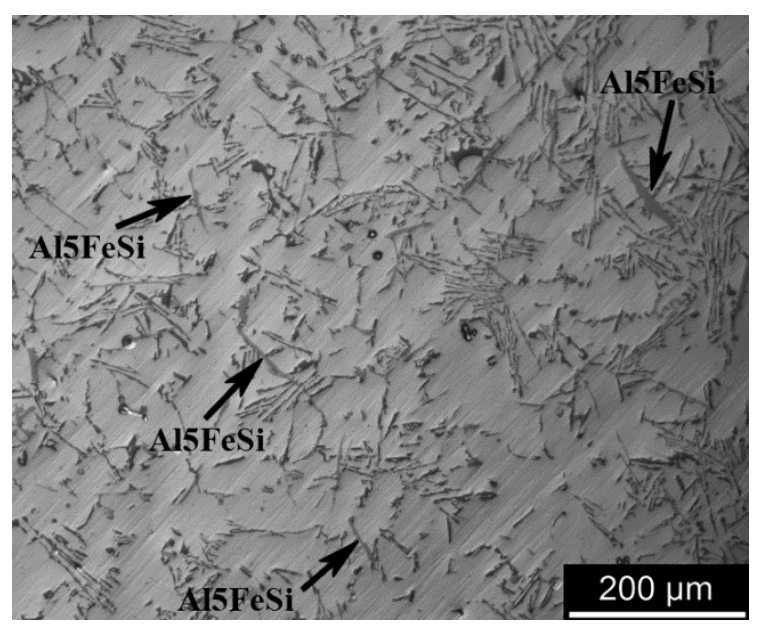


Fig. 9. Microstructure of as-cast AlSi9Cu3 obtained without US processing

\section{Conclusions}

The main goal of this study was the development of a casting technique of Al-Si alloys, to improve the as-cast mechanical properties, based on the use of acoustic energy to perform melt treatment, and analysis of its potential to refine primary a al grain, and to modify the eutectic silicon.

In what concerns to microstructure refining and modification by ultrasound, the main conclusions to be drawn from the experimentation are:

- US processing is an external supply of energy - physical process environmentally clean and efficient that promotes refinement of the $\alpha$-Al and eutectic Si phases, and a decrease of porosity.

- US processing promotes the change of $\alpha$-Al dendritic morphology, which is typical of die-castings, into globular morphology which size depend on the electric power and the distance to the interface of radiation. Globular $\alpha-\mathrm{Al}$ grains with average size of $36 \mu \mathrm{m}$ were obtained for $600 \mathrm{~W}$ electric power for positions $\mathrm{A}$ and $\mathrm{B}$, although for position $\mathrm{C}$ the average value was slightly higher (47 $\mu \mathrm{m})$.

- The results of this work suggest that the mechanism of cavitation, in addition to promoting refinement of the microstructure by heterogeneous nucleation/dendritic fragmentation, is also responsible for changing the morphology of eutectic silicon.

- For the processing conditions of this work the average UTS increased from 220 to $339 \mathrm{MPa}$, strain increased from 0.6 to $2.9 \%$ and the volume of porosity decreased from 1.0 to $0.1 \%$ when compared with non US-treated samples. 


\section{Acknowledgments}

This research was supported by FCT - Portuguese Foundation for Science and Technology and was developed on the aim of the research project PTDC/EMETME/119658/2010 and the Post-Doctoral grant SFRH/BPD/76680/2011. Acknowledgements also to the University of Minho, for the provision of research facilities.

\section{References}

[1] J.E. Gruzleski, B.M. Closset, The Treatment of liquid Aluminum-Silicon Alloys, American Foundryman Society, Des Plaines, IL, USA, 1990.

[2] S. Hegde, K.N. Prabhu, J Mater Sci, 43 (2008) 3009-3027.

[3] A.K. Dahle, K. Nogita, S.D. McDonald, C. Dinnis, L. Lu, Materials Science and Engineering: A, 413-414 (2005) 243-248.

[4] J.A. Spittle, Int J Cast Metal Res, 19 (2006) 210-222.

[5] C. Limmaneevichitr, W. Eidhed, Materials Science and Engineering: A, 349 (2003) 197-206.

[6] L. Xiangfa, Y. Lina, L. Jianwen, W. Zhenqing, B. Xiufang, Materials Science and Engineering: A, 399 (2005) 267-270.

[7] W. Khalifa, Y. Tsunekawa, M. Okumiya, Int J Cast Metal Res, 21 (2008) 129-134.

[8] X. Jian, H. Xu, T.T. Meek, Q. Han, Mater Lett, 59 (2005) 190-193.

[9] H.K. Feng, S.R. Yu, Y.L. Li, L.Y. Gong, J Mater Process Tech, 208 (2008) 330-335.

[10] H. Xu, Q. Han, T.T. Meek, Materials Science and Engineering: A, 473 (2008) 96104.

[11] G.I. Eskin, Ultrasonic Treatment of Light Alloy Melts, Gordon and Breach Science, Amsterdam, Netherlands, 1998.

[12] H. Puga, J. Barbosa, E. Seabra, S. Ribeiro, M. Prokic, Mater Lett, 63 (2009) 806808 .

[13] M. Qian, A. Ramirez, A. Das, J Cryst Growth, 311 (2009) 3708-3715. 
[14] J.D. Hunt, K.A. Jackson, J Appl Phys, 37 (1966) 254-257.

[15] M. Prokic, in: European Patent Application EP1238715, 2001.

[16] H. Puga, S. Costa, J. Barbosa, S. Ribeiro, M. Prokic, J Mater Process Tech, 211 (2011) 1729-1735.

[17] X. Jian, T.T. Meek, Q. Han, Scripta Mater, 54 (2006) 893-896.

[18] S.L. Zhang, Y.T. Zhao, X.N. Cheng, G. Chen, Q.X. Dai, J Alloy Compd, 470 (2009) 168-172.

[19] D. Sunnel, D. Nageswara, C. Satyanarayana, J. Pawan, in: AIJSTPME, 2009, pp. 53-60.

[20] H. Tanaka, S. Shimada, N. Ikawa, P I Mech Eng C-J Mec, 218 (2004) 583-590.

[21] M. Panuskova, E. Tillova, M. Chalupova, Strength of Materials, 40 (2008) 98-101.

[22] Y.M. Li, R.D. Li, Science and Technology of Advanced Materials, 2 (2001) 277280 .

[23] L. Backerud, G. Chai, J. Tamminen, Solidification Characteristics of Aluminum Alloys, AFS/SKANALUMINIUM, Stokholm, Sweden, 1990. 